\title{
The Effects of Material Interfaces on Thermal Resistance Values for High-Power Microwave Transistors
}

\author{
Eric M. Johnson, Peter H. Aaen, Daren Bridges, and John Wood* \\ Freescale Semiconductor, Inc., RF Division, Tempe, AZ
}

\begin{abstract}
In this paper, we examine the effects that the thermal interface between materials has on the thermal resistance extracted from electrical measurements. We also examine the consequent degradation in electrical performance. We present the characterization of the transistor under various thermal environments: mounted in packages, using various die attachments, and on-wafer. A wide range of thermal resistances are extracted and we demonstrate the importance of proper characterization during on-wafer thermal measurements. Finiteelement simulations show that we can account for various thermal interfaces by changing the coefficient of thermal transfer from the semiconductor device to the material beneath it. Simulations are shown to be in good agreement with measured results.
\end{abstract}

\section{INTRODUCTION}

In high-power wireless infrastructure applications, the development of high-efficiency power amplifiers is essential to the cost-effective operation and environmental friendliness of base-stations. Transistors used in these applications are capable of generating several hundred watts of power at microwave frequencies and consequently generate significant amounts of heat.

The amount of heat generated is determined from the definition of power-added efficiency (PAE) computed by

$$
P A E=\frac{P_{\text {out }}-P_{\text {in }}}{P_{d c}}
$$

where $P_{\text {in }}$ and $P_{\text {out }}$ are the input and output power at the operational frequency of the transistor, respectively, and $\mathrm{P}_{\mathrm{dc}}$ is the supplied DC-power. Power which is not converted from $\mathrm{DC}$ to RF or input into the device, approximately

$$
P_{\text {diss }}=P_{d c}(1-P A E)
$$

is dissipated as heat. The generated heat flows away from the transistor junction through the semiconductor and down into the package flange and heat sinks [1].

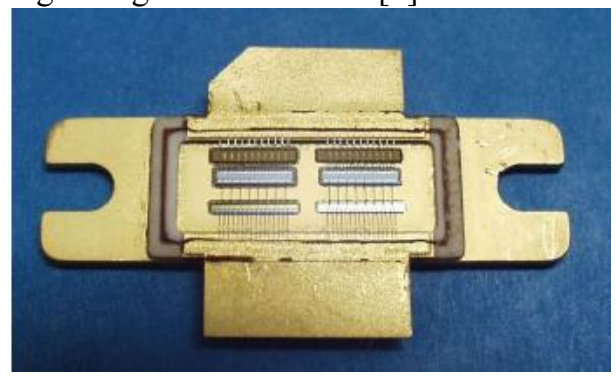

Fig. 1. A high-power LDMOS microwave transistor (courtesy of Freescale Semiconductor, Inc.).

Increased temperatures of operation degrade a transistors performance and impact reliability and therefore there is intensive development into new packaging methodologies and materials [2]. While much of the package development is focused on reliable operation of the transistor, it is essential for the modeling engineer to include self-heating effects within the transistor model. Furthermore, the environment in which the transistor model is extracted must also be carefully considered.

Compact models for high power RF transistors are frequently derived from devices much smaller than those used in the end product. This is primarily due to the difficulties encountered in characterizing a large-periphery high power RF transistor. These include: high currents and power dissipation; oscillations under DC conditions as large periphery devices may be unstable in a $50 \Omega$ measurement system; and the transistor impedances may be too low to make measurements of sufficient accuracy for model extraction. Additionally, the measurements used for model extraction are often performed on-wafer. While such a configuration lends itself well to full device characterization, especially when pulsed measurements are employed, we will show that the thermal characteristics can be significantly different from those of a practical power transistor.

A well-extracted transistor model derived from isothermal measurements can be accurately scaled to a larger periphery device, provided that those measurements are also made under isothermal (or nearly isothermal) conditions. In order to properly model operation under continuous wave or other measurement conditions where a significant amount of heat is dissipated, thermal effects must be taken into account. This is commonly done by including a thermal sub-circuit as part of the transistor non-linear model. For Freescale Semiconductor's FET $^{2}$ model, a thermal sub-circuit consisting of a parallel RC network is used to de-rate the drain current as the channel temperature increases [3].

The impact of thermal resistance $\left(\mathrm{R}_{\mathrm{th}}\right)$ on transistor performance degradation can be illustrated by comparing DC and RF simulations of the same transistor model with significantly different $R_{\text {th }}$ values. Figure 2 shows the IV curves and dynamic load-lines for a device with a total gate width of $9.6 \mathrm{~mm}$, while Figure 3 shows the corresponding PAE vs. $P_{\text {in }}$ curve for the same device model. In both plots, the $\mathrm{R}_{\text {th }}$ was set to values which were determined by the same technique, but for different thermal interfaces. 


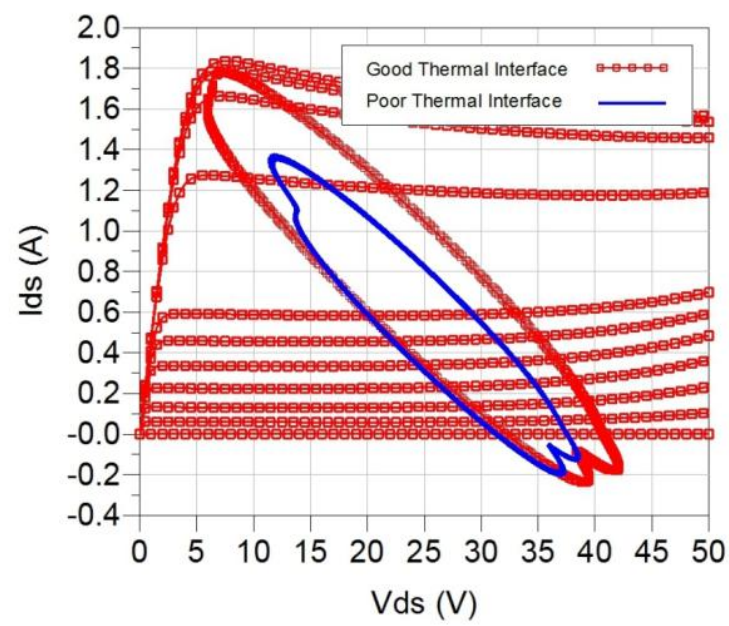

Fig. 2. Simulated $I_{d}-V_{d}$ curves superimposed with dynamic loadlines for a transistor model using two $\mathrm{R}_{\text {th }}$ values, representing good and poor thermal interfaces.

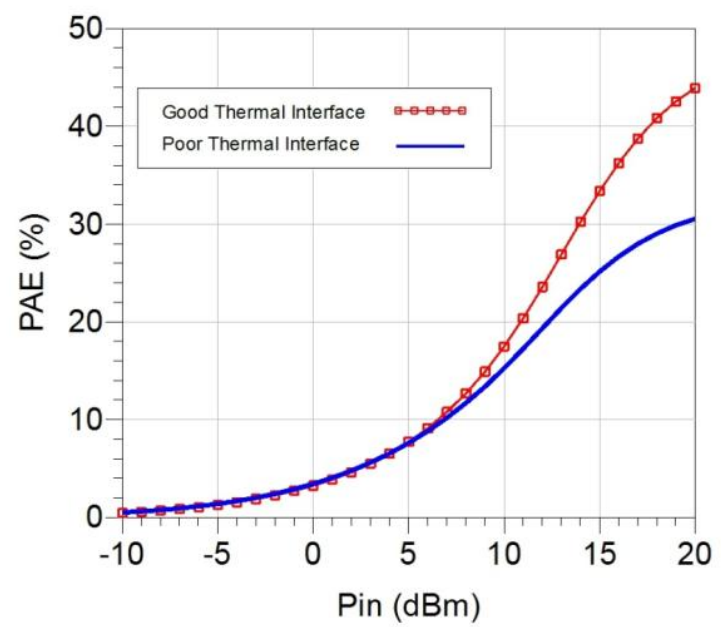

Fig. 3. Simulated power added efficiency curves for a transistor model using two $\mathrm{R}_{\mathrm{th}}$ values, representing good and poor thermal interfaces.

In this paper we investigate the effects of various die attachment methods on the thermal behavior of the transistor. Careful consideration is given to the measurement process and results are correlated with measurement. In the Sections that follow, we shall present a background on methods for thermal resistance measurement using infrared (IR) microscopy and techniques based on electrical measurements that enable the calculation of $R_{t h}$. We will show the sensitivity of the electrical parameters to various interfaces with the semiconductor die and present a finite-element based analysis used to correlate with measured results.

\section{DETERMINATION OF THERMAL RESISTANCE IN DIFFERENT ENVIRONMENTS}

Thermal resistance can be related to the power dissipation of a device by the equation

$$
T_{c}=T_{a}+R_{t h} \times P_{\text {diss }}
$$

where $T_{c}$ is the channel temperature of the device, $T_{a}$ is the ambient temperature, $\mathrm{P}_{\text {diss }}$ is the power dissipated by the device, and $R_{t h}$ is the thermal resistance of the device. In order for the thermal performance to be accurately predicted, the correct value of thermal resistance must be used. Multiple methods exist for determining the thermal resistance for a specific device. Two of the most common methods employ electrical or infrared microscopy techniques. No matter which method is used to determine the thermal resistance, the resulting $\mathrm{R}_{\text {th }}$ can then be scaled along with other model parameters to model larger periphery devices.

One electrical measurement technique employs pulsed measurements. If the device is operated with sufficiently short pulses, the electrical characteristics can be measured while the channel temperature is effectively the same as the ambient temperature. Comparing these isothermal measurements with DC measurements allows for correlation of channel temperature and dissipated power, and the $\mathrm{R}_{\text {th }}$ value can be easily determined [4].

Infrared microscopy is another technique that can be used to measure the $\mathrm{R}_{\mathrm{th}}$ of a device directly. With the device biased at a fixed power dissipation and the ambient temperature known, infrared thermal scanning equipment can be used to measure the temperature of the die surface. The main limitations of this technique are the relatively coarse spatial resolution and the fact that it is the channel temperature, rather than the die surface temperature, that is being measured [4], [5].

A third approach to determine the thermal resistance values uses finite-element based thermal simulations. Recent work in this area has highlighted the use of accurate, rapidly-computed thermal resistance matrices derived from finite-element thermal simulations that take advantage of the transistors repetitive geometry to speed up the computation, and show good correlation with measured results. Figures 4 and 5 show plots of temperature distribution across the surface of a LDMOS transistor, and the measured and simulated temperatures along two cutlines of the temperature distribution plot [5]. 


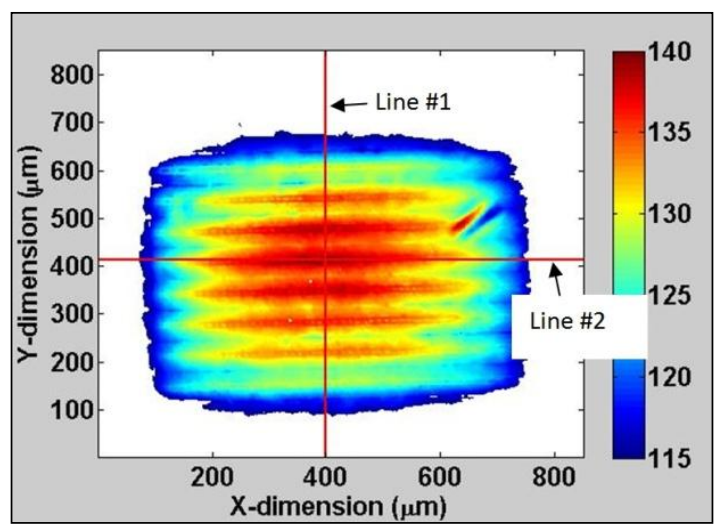

Fig. 4. A plot of the temperature distribution across the surface of an LDMOS transistor under DC operation, 9.6 W of dissipated power. The numbered lines indicate the location of the temperature profiles for Fig. 5

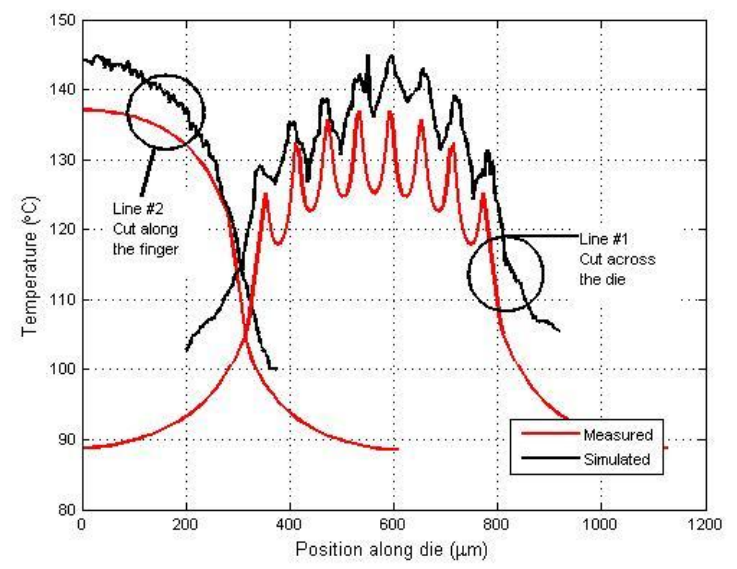

Fig. 5. Measured and simulated temperature plots along lines \#1 and \#2 as shown in Fig. 4.

The electrical measurement techniques described earlier was used to extract the thermal resistance of two devices of the same size and same technology: one on-wafer, the other in-package. The devices used had a total gate width of 9.6 $\mathrm{mm}$, with sixteen fingers of $600 \mu \mathrm{m}$ unit gate width. The onwafer extraction employed measurements using groundsignal-ground probes and a thermal temperature chuck. For the second configuration, a discrete version of the die was attached to a metal carrier using a eutectic die attach process, and then wire-bonded to transmission-line launches. The packaged part was then mounted in a test fixture, with a layer of thermal grease applied to the bottom of the carrier to ensure a good thermal interface between the package and the fixture. The resulting extracted thermal resistances differ by a factor of 7.3, with the thermal resistance extracted from on-wafer measurements having the larger value. This large delta in thermal resistance at first seems difficult to explain, until we use finite-element thermal simulations to examine the source of this discrepancy more closely.

\section{FINITE-ELEMENT THERMAL ANALYSIS}

As previously described, finite-element thermal simulations, such as ANSYS ${ }^{\mathrm{TM}}$, can quickly produce accurate results that are in good agreement with IR microscopy results. Within the ANSYS environment, the option exists to specify the heat transfer coefficient, $H_{c}$. This coefficient, along with temperature, is used to define the boundary condition within ANSYS. Lower values of $\mathrm{H}_{\mathrm{c}}$ result in less heat transfer across the boundary, while higher values of $\mathrm{H}_{\mathrm{c}}$ result in more efficient heat transfer across the boundary. To investigate the on-wafer $\mathrm{R}_{\text {th }}$ extraction, ANSYS simulations were run for the full device while varying $\mathrm{H}_{\mathrm{c}}$ from 0.05 to 1 . The results are shown in Figures 6 and 7.

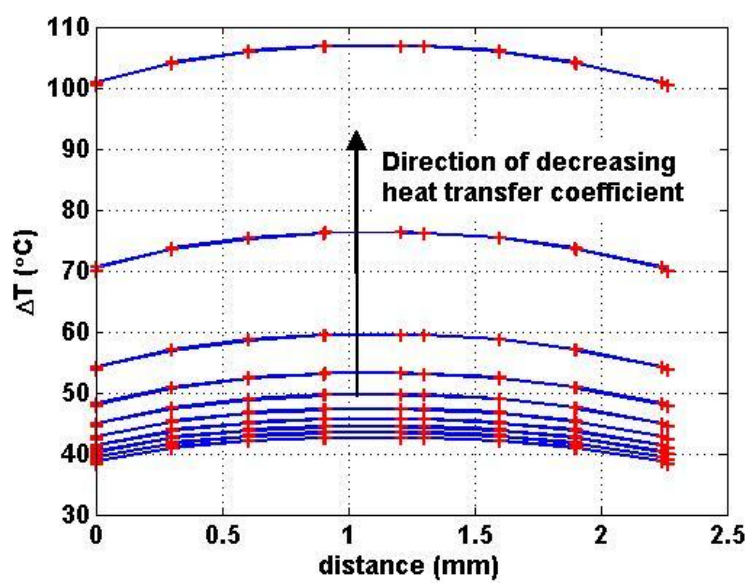

Fig. 6. Simulated temperature rise across a LDMOS transistor as the heat transfer coefficient, $H_{c}$, is varied. As the heat transfer coefficient is decreased, the amount of heat dissipated by the transistor increases rapidly.

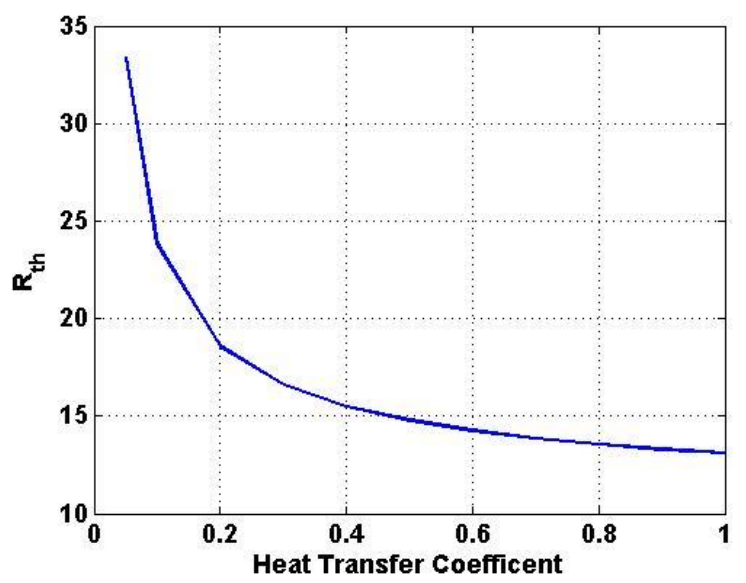

Fig. 7. Extracted thermal resistance from simulation as the heat transfer coefficient is varied.

From Figure 7 , we see that the extracted $R_{\text {th }}$ rises exponentially as $\mathrm{H}_{\mathrm{c}}$ decreases. The $\mathrm{R}_{\text {th }}$ value of $32{ }^{\circ} \mathrm{C} / \mathrm{W}$ extracted from on-wafer electrical measurements matches well with the simulated results for an $\mathrm{H}_{\mathrm{c}}$ value of $\sim 0.06$. The poor thermal interface present in the on-wafer setup is due to the 
surface roughness of the chuck, which effectively adds a layer of air between the chuck and the backmetal of the die. An alternative approach could be to use finite-element thermal simulations to determine the effective thermal transfer coefficients from one material to another. This could be accomplished by optimizing the thermal transfer coefficient until the extracted thermal resistance is the same as that obtained from measurements.

\section{FURTHER MEASUREMENTS AND VALIDATION}

To obtain additional data points, the thermal interface was changed for both the on-wafer and the packaged devices. For on-wafer extraction, a section of wafer had thermal grease applied to the backside of the wafer to improve the thermal interface between the wafer and the chuck. The electrical measurements were repeated for this configuration and the resulting $R_{t h}$ was approximately half that of the value extracted when the bare wafer was held down only by vacuum pressure.

For the packaged part case, a second discrete die was packaged in the same manner as used previously, except that solder was used to attach the die to the metal carrier, instead of a eutectic die attach process. Because of the backmetal properties of the die, we know that attaching the die with solder results in voiding between the die and the metal carrier. The transistor is fully functional, but the thermal interface will be inferior to the case where eutectic die attach process was used. The resulting $R_{t h}$ extracted from this device is approximately the same as that of the original on-wafer measurements with vacuum hold-down. As expected, acoustic microscope scans confirmed the presence of voids under the die; as shown in Figure 8. Conversely, die that were attached using the eutectic process showed little or no voiding, as shown in Figure 9.

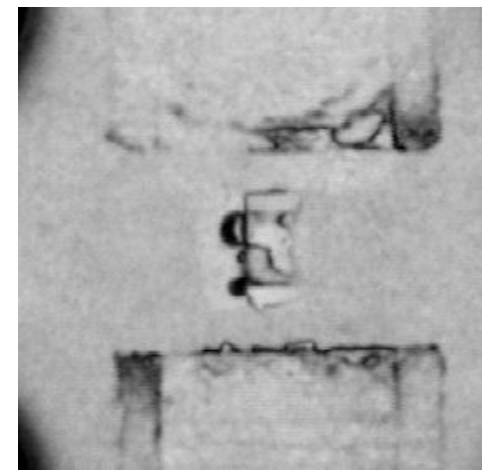

Fig. 8. Scanning acoustic microscope image of a die attached to a carrier with solder. Voiding caused by the poor die attach is clearly visible.

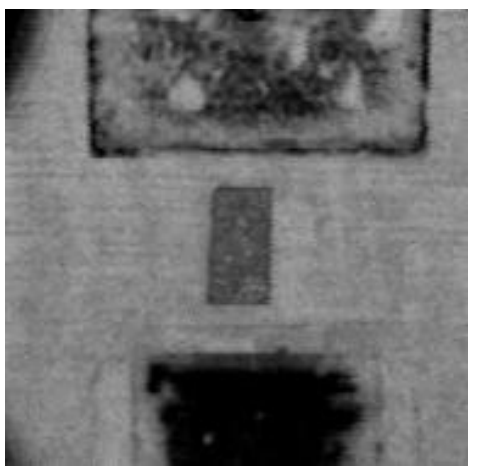

Fig. 9. Scanning acoustic microscope image of a die attached to a carrier by a eutectic die attach process. No voiding under the die is visible.

The thermal resistance values extracted from the four separate sets of electrical measurements are compared in the Table I, along with the value obtained from IR microscopy measurements on a packaged part with eutectic die attach. It should be noted that the lowest thermal resistance value was extracted from the packaged device with eutectic die attach, and that value closely matched the value obtained from finiteelement thermal simulations, and in measurements, the device could be biased to higher currents and power dissipation levels without damaging the transistor. Additionally, $I_{d}-V_{d}$ plots are shown for the various measurement environments. The pulsed $I_{d}-V_{d}$ curves for each device are similar, but the large variation in thermal resistance causes significant variation in the static DC $\mathrm{I}_{\mathrm{d}}-\mathrm{V}_{\mathrm{d}}$ curves.

Table I

Thermal resistances extracted from electrical measurements for a 9.6 mm LDMOS transistor in different measurement environments. Thermal resistance extracted using IR microscopy is also included for comparison.

\begin{tabular}{|c|c|}
\hline Measurement Conditions & $\begin{array}{c}\text { Extracted } \mathrm{R}_{\text {th }} \\
\left({ }^{\circ} \mathrm{C} / \mathrm{W}\right)\end{array}$ \\
\hline On-wafer device with vacuum hold-down & 33 \\
\hline On-wafer device with thermal grease & 17 \\
\hline Packaged device with solder die attach & 32 \\
\hline $\begin{array}{c}\text { Packaged device with eutectic die attach } \\
\text { die attach }\end{array}$ & 4.5 \\
\hline $\begin{array}{c}\text { IR microscopy of packaged device with eutectic } \\
\text { mich }\end{array}$ & 5 \\
\hline
\end{tabular}




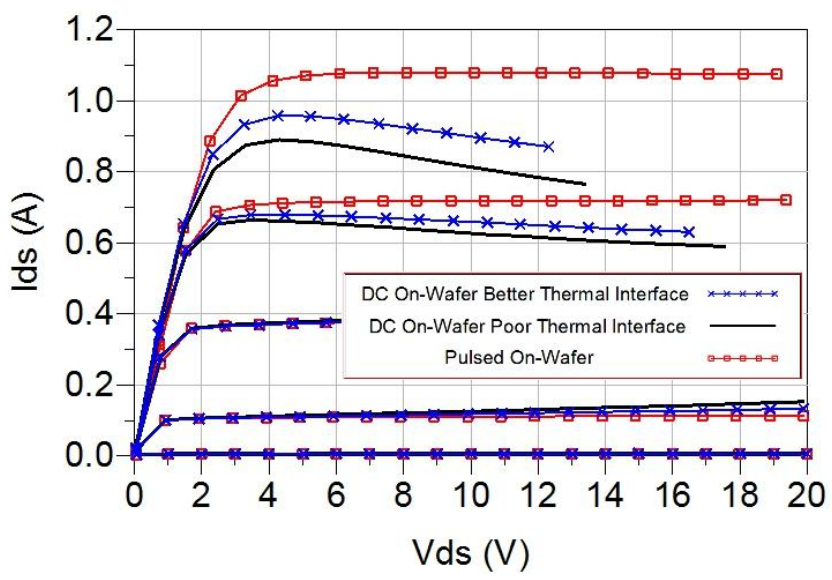

Fig. 10. Comparison of on-wafer $\mathrm{I}_{\mathrm{d}}-\mathrm{V}_{\mathrm{d}}$ measurements. Pulsed (isothermal) measurements are shown along with DC measurements made with a poor thermal interface (bare wafer held down by vacuum), as well as an improved thermal interface (thermal grease between wafer and chuck).

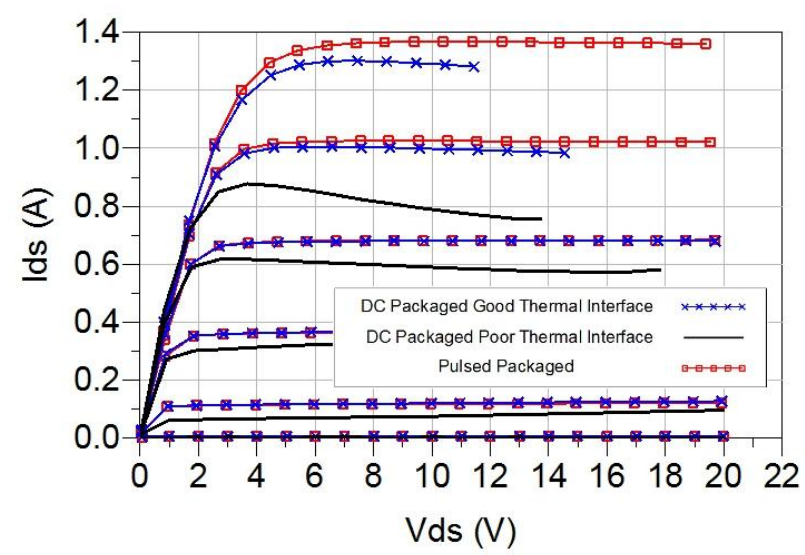

Fig. 11. Comparison of packaged $\mathrm{I}_{\mathrm{d}}-\mathrm{V}_{\mathrm{d}}$ measurements. Pulsed (isothermal) measurements are shown along with DC measurements made with a poor thermal interface (solder die attach, significant voiding) and good thermal interface (eutectic die attach, no voiding).

\section{CONCLUSIONS}

We have shown the importance of properly characterizing the thermal resistance of a RF power transistor for the specific environment that it is used in. Large discrepancies in thermal resistance for the same device in different thermal environments were explained by taking into account the heat transfer coefficient for each environment. The effects of various die attach methods and their impacts on the thermal behavior of a RF power transistor were described. Care must be taken to use the proper method of die attach, or thermal and electrical results may be significantly worse than expected. As a consequence of this, you may wind up optimizing other parameters to compensate for this effect.

\section{ACKNOWLEDGEMENTS}

The authors would like to acknowledge Eddie Mares for assistance with IR microscopy measurements.

\section{REFERENCES}

[1] P.H. Aaen, J.A. Plá, and J. Wood, Modeling and Characterization of $R F$ and Microwave Power FETs. Cambridge, UK: Cambridge University Press, 2007.

[2] Z. Radivojevic, K. Andersson, L. Bogod, M. Mahalingam, J. Rantala, and J. Wright, "Novel materials for improved quality of RF-PA in base station applications," IEEE Trans. Compon. Packag. Technol., vol. 28, no. 4, pp. 644-649, Dec. 2005.

[3] J. Wood, P. H. Aaen, D. Bridges, D. Lamey, M. Guyonnet, D. S. Chan, and N. Monsauret, "A nonlinear electro-thermal scalable model for high-power LDMOS transistors," IEEE Trans. Microw. Theory Tech., vol. 57, no. 2, pp. 282-292, Feb. 2009.

[4] R. Menozzi, A.C. Kingswood, "A New Technique to Measure the Thermal Resistance of LDMOS Transistors" IEEE Trans. On Device and Materials Reliability, vol. 5, no. 3, pp. 515-521, September 2005.

[5] P.H. Aaen, J. Wood, Q. Li, and E. Mares, "Thermal resistance modeling for the electrothermal layout of high-power RF transistors," IEEE MTT•S Int. Microwave Symp. Dig., May 2010, pp. 1672-1675. 